\title{
Histochemical Analysis of Lacrimal Concretions in a Patient Using Rebamipide Ophthalmic Suspension: Case Report
}

\author{
Ken Yamashita ${ }^{1} \cdot$ Miki Hiraoka $^{2}$ (D) $\cdot$ Shintaro Sugita ${ }^{3} \cdot$ Takatoshi Yotsuyanagi $^{1}$
}

Accepted: 4 February 2021 / Published online: 15 February 2021

(C) The Author(s) 2021

\begin{abstract}
Concretions of the lacrimal drainage system can cause dacryocystitis. In the present study, our patient developed dacryocystisis with lacrimal concretions of a white soft mass. Rebamipide ophthalmic suspension had been applied for treatment of dry eye. To evaluate the pathogenic mechanism of the case, histological and chemical studies were performed. Our case was woman in her seventies. She had a medical history of rheumatoid arthritis. She was referred to our hospital for dacryocystitis after showing a poor response to treatment with antibiotic agents. A head computed tomography (CT) scan showed ductal high-density deposits along the lacrimal sacs. During dacryocystotomy, the hypertrophy of the lacrimal sacs was found replete with pus and cottage cheese-like white substances. The extracts were surgically removed, and histological and chemical analysis was performed. The histological examination showed granulation tissues and acellular amorphous material with crystal-like structures. Positive staining by Alcian blue and Kossa was found in crystal-like legions. The extract obtained from the concretions showed the same fluorescence band and UV absorption spectrum in thin layer chromatography (TLC) and spectrometry, respectively, as rebamipide. Our findings suggest that the obstruction by lacrimal concretions containing rebamipide resulted in dacryocystitis in this case. In addition, it is speculated that in patients who have impaired tear secretion, refractory dacryocystitis may be evoked following the accumulation of rebamipide in the lacrimal sacs.
\end{abstract}

Keywords Lacrimal concretion · Dacryocystitis · Rebamipide · Thin layer chromatography $\cdot$ Case report

\section{Introduction}

The lacrimal concretions can develop in any lesion of the lacrimal pathway including the canaliculus, lacrimal sac, and nasolacrimal duct. The lacrimal concretions have also been termed as dacryoliths, mucoliths, and/or canaliculolith. It can lead to obstruction of the tear passage and cause dacryocystitis. The region of dacryocystitis is frequently accompanied with infection of the fungus and Actinomyces [1].

This article is part of the Topical Collection on Medicine

Miki Hiraoka

mikihira@nms.ac.jp

1 Department of Plastic and Reconstructive Surgery, Sapporo Medical University School of Medicine, Sapporo, Hokkaido, Japan

2 Department of Ophthalmology, Health Sciences University of Hokkaido, 2-5-1 Ainosato Kitaku, Sapporo, Hokkaido 002-8072, Japan

3 Department of Surgical Pathology, Sapporo Medical University School of Medicine, Sapporo, Hokkaido, Japan
Tears are indispensable to maintain transparency of the cornea by supplying moisture and nutrients. Insufficient secretion of tears leads to dry eye syndrome. Tears consist of water, proteins, mucins, and lipids. A deficiency of mucins in tears is known to cause dry eye syndrome [2]. Treatment for dry eye syndrome is mainly the administration of eye drops such as a physiological saline solution, sodium hyaluronate solution, diquafosol sodium solution, and lacrimal plugs. Recently, rebamipide ophthalmic suspension (ROS) has been recognized as a new therapeutic eye drop. Rebamipide was originally developed to treat gastric ulcers and gastritis by increasing mucin-like substances and protecting the internal surface of the stomach [3-5]. Rebamipide is hydrophobic particles. ROS has been applied to a large number of dry eye patients with positive outcomes [6]. Among its effect, it increases the number of conjunctival goblet cells, produces a mucin-like substance in corneal epithelial cells, and facilitates wound healing at corneal epithelial cells [7-10]. However, complications such as lacrimal passage obstruction and dacryocystitis have been reported [11].

In this report, we describe the case presenting dacryocystitis with a white substance in the lacrimal sac. Histological 
examination and chemical analysis were carried out to understand the characteristic phenotype and etiology of lacrimal concretions following ROS application.

\section{Case Presentation}

The medical records of the patient with dacryocystitis with foreign substances were retrospectively reviewed. The present study protocol was approved by the Ethics Committee of Sapporo Medical University Hospital and conducted in accordance with the Declaration of Helsinki. After a full explanation of the purpose and protocol for this study, patient provided written informed consent for publication. We followed the CARE Guidelines for case reports study on human subjects.

Our patient was a 77-year-old woman who had developed acute dacryocystitis on her left lid 2 years prior to presentation. She had been previously diagnosed with dry eye and glaucoma. Additionally, she had undergone ROS for a year before the development of dacryocystitis. The ROS application was terminated at the onset of dacryocystitis because it was thought that ROS might have been involved in the formation of dacryocystitis. Moreover, she did not show symptoms of epiphoria at the time. She had initially visited a separate hospital where she received paracentesis and had her lacrimal sac drained, both of which resulting in improvements in her condition. Six months later, the dacryocystitis relapsed and the same treatment was applied, again with a positive outcome. A year later, she developed dacryocystitis on her right lid. Application of an antibacterial agent proved to be effective. However, a fistula between the lacrimal sac and skin was found 3 months after a right lacrimal tube insertion. She had been diagnosed previously with hypertension and rheumatoid arthritis that was being medically controlled.

Examination disclosed an erythematous and swelling area in the right lower lid. Discharge was observed from the right lacrimal point. A head computed tomography (CT) scan demonstrates ductal high-density deposits along the right lacrimal sac (Fig. 1a, b). From the CT image findings, a diagnosis of dacryocystitis with dacryoliths was reached. Under systemic anesthesia, a dacryocystotomy was performed, and the incisions were sutured after the contents were extracted. The lacrimal sac was hypertrophic and inundated with pus and calcified white substances. A histological study and chemical analysis were conducted for those extracts. There was no relapse of dacryocystitis after surgery.

\section{Histological Analysis}

The extracts showed a cottage cheese-like white mass and granular tissues. Extracts were stored in formalin and then embedded in paraffin. Hematoxylin and eosin (HE) staining, Alcian blue staining, Kossa staining, and Grocott staining were then performed on the samples. The findings of HE staining showed inflammatory granulation tissues with severe infiltration of lymphocytes, plasma cells, and neutrophils. Also, Actinomyces colonies are observed (Fig. 2e). Additionally, there is a crystal-like structure (Fig. 2a) surrounded by abscesses in all cases. In the crystal-like structure, a diffuse positive pattern with Alcian blue and Kossa staining is found (Fig. 2b, c). In granulation tissues, positive staining is partially observed by Alcian blue and Kossa staining (Fig. 2d).

\section{Chemical Analysis of Lacrimal Concretions}

According to the chemical structure of rebamipide, it is a lipophilic small molecule. Five microliter of the rebamipide suspension (20 $\mathrm{mg}$ rebamipide/ml, Otsuka Pharmaceutical Co., Tokyo Japan) was suspended with $1 \mathrm{ml}$ of $0.9 \% \mathrm{NaCl}$, mixed with $2 \mathrm{ml}$ of methanol plus $2 \mathrm{ml}$ of chloroform, and centrifuged at $800 \mathrm{~g}$ for $5 \mathrm{~min}$ at room temperature. The resultant organic phase was transferred into another glass tube and dried down under a stream of nitrogen gas. The dried extract was dissolved with $100 \mu \mathrm{l}$ of chloroform/methanol $(2: 1, \mathrm{v} / \mathrm{v})$. A part of the extract was applied to an HPTLC plate and developed in a solvent system consisting of chloroform/methanol/ water $(60: 35: 8, \mathrm{v} / \mathrm{v})$. To visualize rebamipide, the plate was dried and then exposed under ultraviolet (UV) light. The same extract was then dried again and dissolved with dimethylformamide (DMF). The absorption spectrum of rebamipide in DMF was recorded within the scanning range $270-400 \mathrm{~nm}$ using a spectrophotometer (Hitachi U-3010 spectrophotometer, Japan). To identify rebamipide in the concretion, $21 \mathrm{mg}$ (wet weight) of the clinical sample was homogenized with $0.8 \mathrm{ml}$ of cold $0.9 \% \mathrm{NaCl}$ for 20 strokes using a 1-ml Teflon-glass homogenizer on ice. The homogenates $(100 \mu \mathrm{l})$ were transferred into a glass tube, mixed $0.7 \mathrm{ml}$ of $0.9 \% \mathrm{NaCl}$ plus $2 \mathrm{ml}$ of methanol and $1 \mathrm{ml}$ of chloroform, briefly sonicated by a sonic bath, and stood for $30 \mathrm{~min}$ at room temperature. The suspensions were then mixed with $1 \mathrm{ml}$ of chloroform plus $0.2 \mathrm{ml}$ of $0.9 \% \mathrm{NaCl}$ and centrifuged at $800 \mathrm{~g}$ for $5 \mathrm{~min}$ at room temperature. The resultant organic phase was transferred into another glass tube and dried down under a stream of nitrogen gas. The dried materials were examined as the extract obtained from the rebamipide suspension.

The concretion obtained from the patient was a white and water-insoluble soft lump. Rebamipide is a water-insoluble white powder. As shown in the thin layer chromatography 
Fig. 1 Head computed tomography $(\mathrm{CT})$ scan. The born window image on the coronal section (a) and the contrastenhanced image on the axial section, (b) demonstrated highdensity deposits in right lacrimal $\mathrm{sac}$

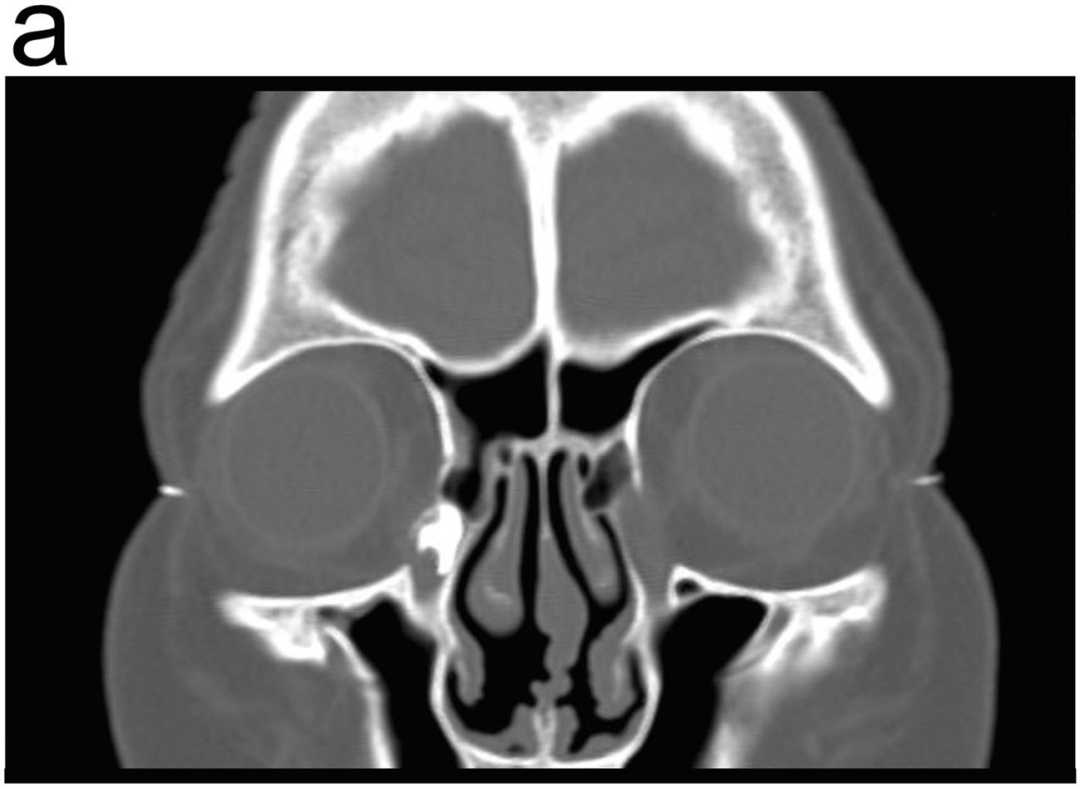

b

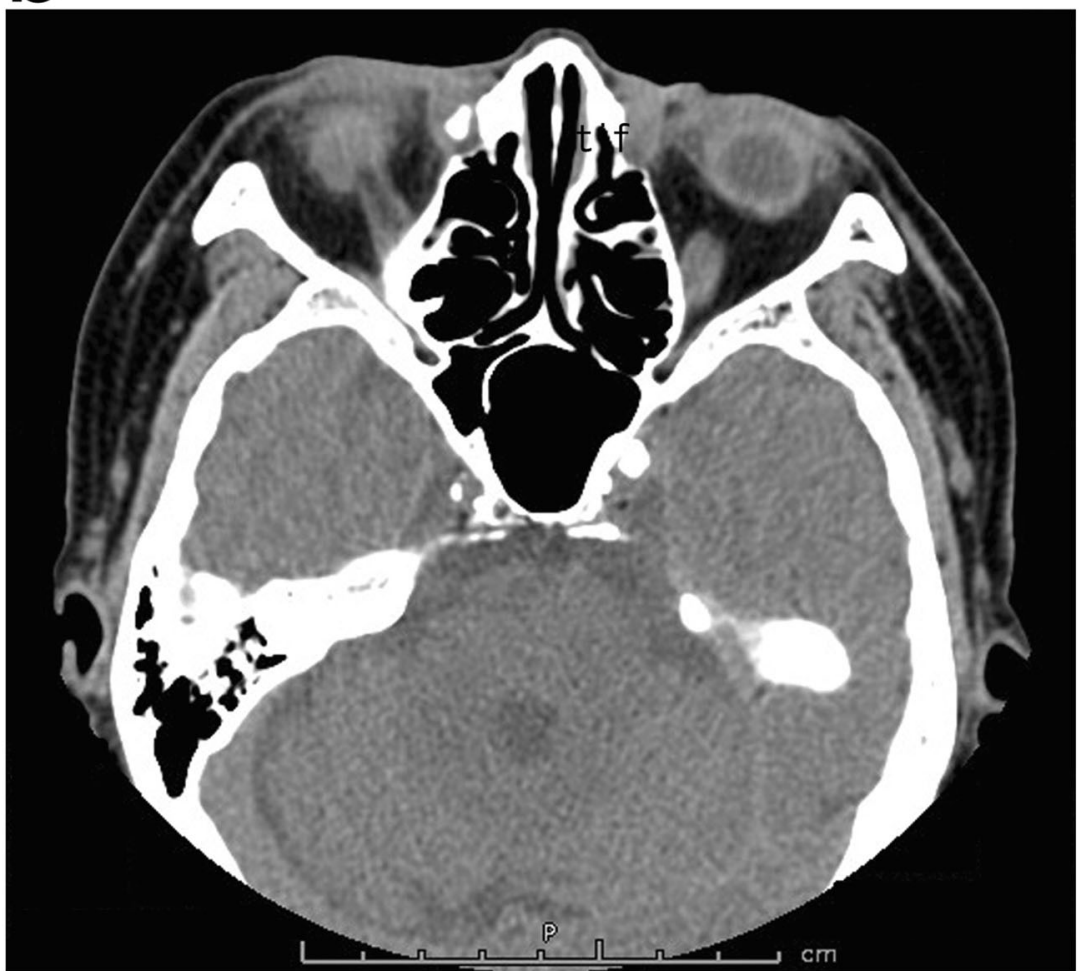

(TLC), the rebamipide extracted from the ophthalmic suspension is detected as a single band with fluorescence emission by UV light exposure (Fig. 3a). In addition, the rebamipide in DMF shows a characteristic UV absorption spectrum (Fig. $3 b)$. Intriguingly, the extract obtained from the concretion showed the same fluorescence band and UV spectrum in TLC and spectrometry, respectively, as rebamipide. These results show that the concretion contained water-insoluble ingredient rebamipide.

\section{Discussion}

In this report, we described the case of dacryocystitis containing concretions. Our case had a history of using ROS. The histological study showed a crystal-like structure in specimens. It was assumed these substances formed crystal-like structure contained unbiological materials that caused the concretions. Therefore, we considered the possibility that the white substance found in the lacrimal path and/or sacs of the 
a

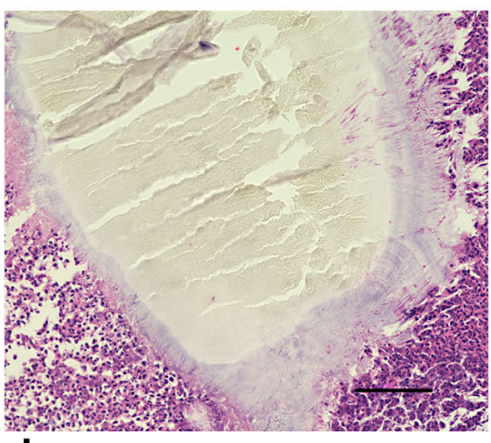

d

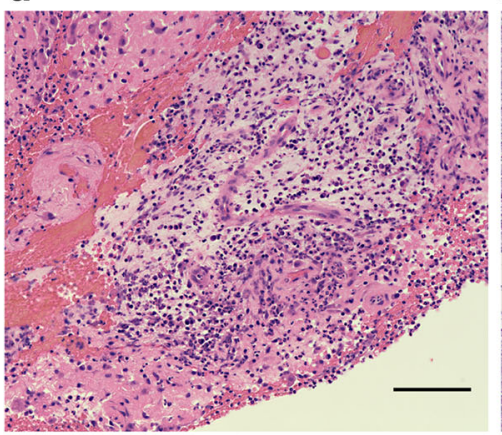

b

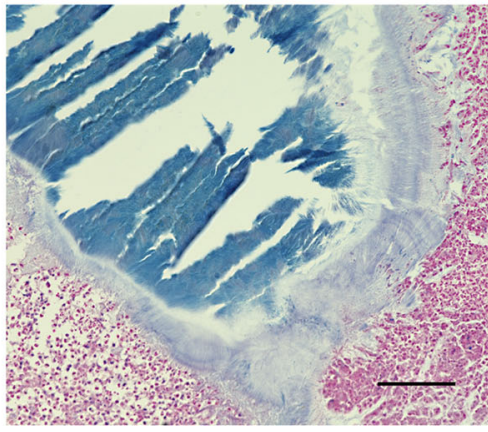

e

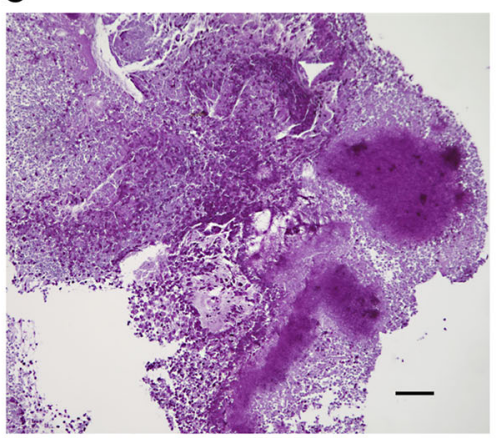

C

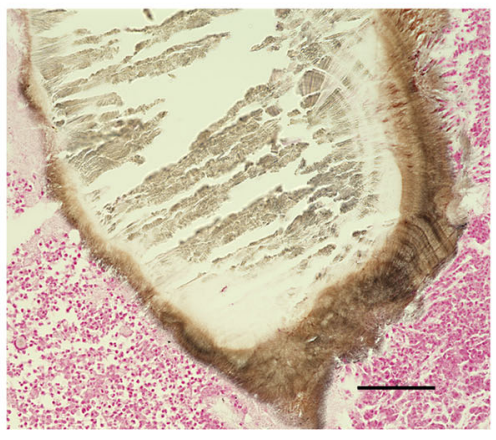

lesion of the crystal-like structure area. $(\mathbf{d}, \mathbf{e})$ The lesion of the granulation area. Scale bar indicates $100 \mu \mathrm{m}$
Fig. 2 Histology of surgically removed specimen. It showed crystal-like structure and granulation tissues. (a, d) Hematoxylin-eosin staining. (b) Alcian blue staining, (c) Kossa staining, (e) Grocott staining. (a, b, c) The patient was formed or developed by accumulation of rebamipide. Results of the chemical analysis of the specimen did in fact reveal that the concretion contained rebamipide.

Dacryocystitis is inflammation of the lacrimal sac. The causes of csytitis vary and include infection, autoimmune diseases, and malignant diseases [12]. The majority is induced by infection. Our case showed colonies of acetinomysis. Acetinomysis is a type of indigenous bacterium in the mouth and is frequently found in dacryocystitis. The histological study in our case showed granular tissues and crystal-like a

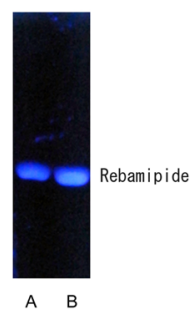

b

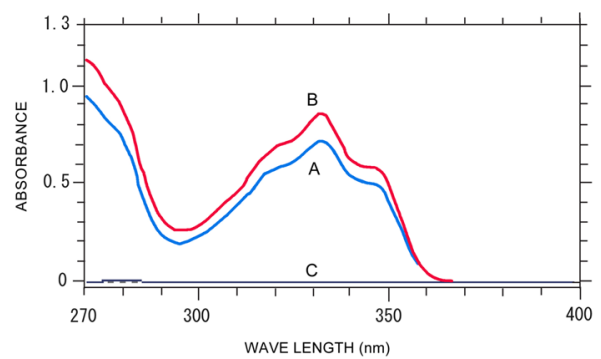

Fig. 3 Chemical analysis of white mass A. (a) In thin layer chromatography (TLC), white mass extracts (B) showed the same mobility as rebamipide (A). (b) In UV spectrometry, white mass extracts (B) showed the same spectrum pattern as rebamipide (A). Negative control (DMF) did not show any peak (C) structures. In the granulation tissue area, non-uniform patterns were found through Alcian blue staining. It is difficult to confirm whether the positive staining was caused by rebamipide application or responses to inflammation. The screening of other dacryocystitis specimens without rebamipide application is required to answer this question. There was a faint stain at the marginal region by Kossa staining. However, at the crystal-like structure area, there was prominent staining by Alcian blue and Kossa. It is likely that the positive staining by Alcian blue reflects the mucin augmentation.

Previously, we had experienced other 2 cases of dacryocystitis after showing a poor response to treatment with antibiotic agents [13]. A head CT scan revealed ductal highdensity deposits along the bilateral lacrimal sac. It is assumed that these types of high-density deposits reflect the accumulation of ROS accumulation. Both cases had medical history of rheumatoid arthritis with Sjögren syndrome and had been applied ROS for dry eye treatment. Dacryocystotomy was performed, and the contents were extracted. Histological study showed granulation tissues with Actinomyces colonies and crystal-like structure. Interestingly, all three cases were incidentally affected by rheumatoid arthritis. It is difficult to ascertain whether this systemic background had any influence on the patients contracting dacryocystitis with concretions. It can be speculated that an aggressive autoimmune response following rheumatoid arthritis leads to inflammation and 
granular tissue formation in the lacrimal pathway. Another possibility is that the immunosuppressive state for the rheumatoid arthritis treatment precedes susceptibility to infections such as Actinomyces. We conjecture that the low tear secretion results in a disturbance of the washout system to remove unnecessary substances in the lacrimal pathway.

From the outcome of those 3 cases (the present case and previous 2 cases), it is presumed that ROS application is involved in the development of dacryocystitis. However, it is difficult to distinguish whether the ROS leads the obstruction of the lacrimal pathway or rather accelerates the obstruction in addition to the nasolacrimal duct stenosis. To trace the time course of dacryocystitis formation by dacryocystography from the onset of lacrimal pathway obstruction may clarify the query.

The content of dacryoliths was found to be organic material with minerals such as calcium and magnesium [14]. In addition, Mano et al. reported that the protein profiles of dacryoliths were different between ROS and non-ROS group [15]. However, the exact composition of organic material is unclear in either with or without ROS application.

The chemical analysis of the specimen in our case revealed that the water-insoluble ingredient contained rebamipide. It is assumed that the crystal-like structure found during histological examination included rebamipide. Rebamipide is water insoluble. When there is stenosis of the lacrimal pathway, the aggregation of rebamipide can obstruct the lacrimal pathway. Then, dacryocystitis can be induced at this site following infection of such as Actinomyces.

In light of these results, it can be inferred that the accumulation of rebamipide particles leads to congestion of the lacrimal pathway and complications of actinomycosis can result in the formation of dacryocystitis. In addition, immunosuppressive disorders such as rheumatoid arthritis and Sjögren syndrome may increase the risk of dacryocystitis under ROS application.

Our results suggest that the ROS should be carefully applied to any patient. It is recommended that when a patient shows the improvement of dry eye symptoms or the development of epiphoria upon the application of ROS, it is recommended to terminate the application because it may be a sign of the lacrimal pathway being obstructed by rebamipide. Also, it is better to confirm this possibility through the use of a head CT scan or dacryocystography. In case of severe dry eye patients such as those suffering from Sjogren syndrome, the epiphoria may not present due to the minor lacrimation. Thus, it is important to be cautious when applying ROS to severe dry eye patients. The ROS application to these patients may result in concretions on the lacrimal pathway and induce dacryocystitis.

Acknowledgments We are grateful to Dr. Akira Abe for giving us instructions for chemical analysis.
Authors' Contributions K.Y. and T.Y. managed the patient clinically. S.S. performed and evaluated histological study. M.H. performed and evaluated biochemical study. M.H., K.Y. and S.S. wrote the manuscript. T.Y. revised draft. All authors discussed the results and approved the final manuscript.

\section{Declarations}

Ethical Approval All procedures performed in this study were in accordance with the ethical standards of the Ethics Committee of Sapporo Medical University Hospital and with the 1964 Helsinki declaration and its later amendments or comparable ethical standards.

Informed Consent Informed consent was obtained from the participant included in the study.

Data availability Statement The data that support the findings of this study are available from the corresponding author, $\mathrm{MH}$, upon reasonable request. However, due to privacy and ethical concerns, the data about patient information is not available.

Conflict of Interest The authors that they have no conflict of interest.

Abbreviations CT, Computed tomography; TLC, Thin layer chromatography; ROS, Rebamipide ophthalmic suspension; HE, Hematoxylin and eosin; UV, Ultraviolet; DMF, Dimethylformamide

Open Access This article is licensed under a Creative Commons Attribution 4.0 International License, which permits use, sharing, adaptation, distribution and reproduction in any medium or format, as long as you give appropriate credit to the original author(s) and the source, provide a link to the Creative Commons licence, and indicate if changes were made. The images or other third party material in this article are included in the article's Creative Commons licence, unless indicated otherwise in a credit line to the material. If material is not included in the article's Creative Commons licence and your intended use is not permitted by statutory regulation or exceeds the permitted use, you will need to obtain permission directly from the copyright holder. To view a copy of this licence, visit http://creativecommons.org/licenses/by/4.0/.

\section{References}

1. Hawes MJ. The dacryolithiasis syndrome. Ophthalmic Plast Reconstr Surg. 1988;4:87-90.

2. Mantelli F, Argueso P. Functions of ocular surface mucins in health and disease. Curr Opin Allergy Clin Immunol. 2008;8:477-83.

3. Kleine A, Kluge S, Peskar BM. Stimulation of prostaglandin biosynthesis mediates gastroprotective effect of rebamipide in rats. Dig Dis Sci. 1993;38:1441-9.

4. Higuchi K, Arakawa T, Nebiki H, Uchida T, Fujiwara Y, Ando K, et al. Rebamipide prevents recurrence of gastric ulcers without affecting Helicobacter pylori status. Dig Dis Sci. 1998;43(9):99S$106 \mathrm{~S}$.

5. Naito Y, Yoshikawa T, Iinuma S, Yagi N, Matsuyama K, Boku Y, et al. Rebamipide protects against indomethacin-induced gastric mucosal injury in healthy volunteers in a double-blind, placebocontrolled study. Dig Dis Sci. 1998;43(Suppl 9):83S-9S.

6. Kinoshita S, Oshiden K, Awamura S, Suzuki H, Nakamichi N, Yokoi N. Rebamipide ophthalmic suspension phase 3 study group. A randomized, multicenter phase 3 study comparing $2 \%$ rebamipide (OPC-12759) with $0.1 \%$ sodium hyaluronate in the treatment of dry eye. Ophthalmology. 2013;120:1158-65. 
7. Urashima H, Okamoto T, Takeji Y, Shinohara H, Fujisawa S. Rebamipide increases the amount of mucin-like substances on the conjunctiva and cornea in the $\mathrm{N}$-acetylcysteine-treated in vivo model. Cornea. 2004;23:613-9.

8. Tanaka H, Fukuda K, Ishida W, Harada Y, Sumi T, Fukushima A. Rebamipide increases barrier function and attenuates TNF $\alpha$ induced barrier disruption and cytokine expression in human corneal epithelial cells. Br J Ophthalmol. 2013;97:912-6.

9. Kimura K, Morita Y, Orita T, Haruta J, Takeji Y, Sonoda KH. Protection of human corneal epithelial cells from TNF- $\alpha$-induced disruption of barrier function by rebamipide. Invest Ophthalmol Vis Sci. 2013;54:2752-60.

10. Kase S, Shinohara T, Kase M, Ishida S. Effect of topical rebamipide on goblet cells in the lid wiper of human conjunctiva. Exp Ther Med. 2017;13:3516-22.

11. Sugimoto Y, Fukuta Y, Tsubota K, Ohashi Y, Kinoshita S. Retrospective review of dacryostenosis, dacryocystitis and foreign body in eye or lacrimal duct under administration of rebamipide ophthalmic susupension (Mucosta ophthalmic suspension UD 2\%). J Eye. 2015;32:1741-7 Japanese.

12. Anderson NG, Wojno TH, Grossniklaus HE. Clinicopathologic findings from lacrimal sac biopsy specimens obtained during dacryocystorhinostomy. Ophthalmic Plast Reconstr Surg. 2003;19:173-6.

13. Ikeda T, Hiraoka M, Inatomi S, Funabashi K, Yamashita K, Sugita S, et al. Bilateral dacryolith in two cases of Sjogren syndrome. Jpn J Clin Ophthalmol. 2017;71:593-8 Japanese.

14. Iliadelis ED, Karabatakis VE, Sofoniou MK. Dacryoliths in chronic dacryocystitis and their compositin (spectrophotometric analysis). Eur J Ophthalmol. 1999;9:266-8.

15. Mano F, Takimoto H, Oe M, Chang K, Mano T, Yoshida Y. Proteomic analysis of dacryoliths from patients with or without topical rebamipide treatment. Biomed Hub. 2018;3:1-11.

Publisher's Note Springer Nature remains neutral with regard to jurisdictional claims in published maps and institutional affiliations. 